\title{
Evidence for Water Rings in the Hexahydrated Sulfate Dianion from IR Spectroscopy
}

Matthew F. Bush, Richard J. Saykally, and Evan R. Williams*

Supplemental Information 


\begin{tabular}{|c|c|c|c|}
\hline \multicolumn{4}{|c|}{$\left[\mathrm{SO}_{4}\left(\mathrm{H}_{2} \mathrm{O}\right)_{6}\right]^{2-}\left(T_{d}\right.$ symmetry $)$} \\
\hline $\mathrm{S}$ & $\odot . \odot \odot \odot \odot \odot \odot$ & $\odot .00 \odot \odot \odot \odot$ & $\odot .00000 \odot$ \\
\hline 0 & $\odot . \odot ० \odot \odot \odot \odot$ & $\odot .00 \odot \odot \odot \odot$ & 1.543227 \\
\hline 0 & 1.454969 & $\odot . \odot \odot \odot \odot \odot \odot$ & $-\odot .514409$ \\
\hline 0 & $-\odot .727484$ & 1.260040 & $-\odot .514409$ \\
\hline 0 & -0.727484 & -1.260040 & $-\odot .514409$ \\
\hline 0 & -1.436722 & -2.488475 & 2.031831 \\
\hline $\mathrm{H}$ & $-\odot .962302$ & -1.666756 & 2.257840 \\
\hline $\mathrm{H}$ & -1.385123 & -2.399104 & 1. 061920 \\
\hline 0 & -2.873444 & $\odot . \odot \odot \odot \odot \odot \odot$ & -2.031831 \\
\hline $\mathrm{H}$ & -2.347425 & 0.732348 & -1.659880 \\
\hline $\mathrm{H}$ & -2.347425 & -0.732348 & -1.659880 \\
\hline 0 & -1.436722 & 2.488475 & 2.031831 \\
\hline $\mathrm{H}$ & -1.385123 & 2.399104 & 1.061920 \\
\hline $\mathrm{H}$ & -0.962302 & 1.666756 & 2.257840 \\
\hline 0 & 2.873444 & $\odot . \odot \odot \odot \odot \odot \odot$ & 2.031831 \\
\hline $\mathrm{H}$ & 2.770246 & ๑. $0000 \odot \odot$ & 1.061920 \\
\hline $\mathrm{H}$ & 1.924604 & $\odot . ๑ \odot \odot \odot \odot \odot$ & 2.257840 \\
\hline 0 & 1.436722 & -2.488475 & -2.031831 \\
\hline $\mathrm{H}$ & 1.807945 & -1.666756 & -1.659880 \\
\hline $\mathrm{H}$ & $\odot .539480$ & -2.399104 & -1.659880 \\
\hline 0 & 1.436722 & 2.488475 & -2.031831 \\
\hline $\mathrm{H}$ & 1.807945 & 1.666756 & -1.659880 \\
\hline $\mathrm{H}$ & $\odot .539480$ & 2.399104 & -1.659880 \\
\hline
\end{tabular}

\section{$\left[\mathrm{SO}_{4}\left(\mathrm{H}_{2} \mathrm{O}\right)_{6}\right]^{2-}\left(C_{3}\right.$ symmetry $)$}

\begin{tabular}{|c|c|c|c|}
\hline 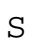 & $\odot . \diamond \odot \odot \odot \odot \odot$ & $\odot . \diamond \odot \odot \odot \odot \odot$ & $\odot .353947$ \\
\hline 0 & 1.352891 & $\odot .531584$ & $-\odot .159097$ \\
\hline 0 & -1.136811 & $\odot .905846$ & $-\odot .159097$ \\
\hline 0 & $\odot . \odot \odot \odot \odot \odot \odot$ & $\odot . \odot \odot \odot \odot \odot \odot$ & 1.896885 \\
\hline 0 & -0.216080 & -1.437430 & $-\odot .159097$ \\
\hline 0 & -0.447755 & -2.857637 & 2.321010 \\
\hline $\mathrm{H}$ & -0.417231 & -2.707240 & 1.355174 \\
\hline $\mathrm{H}$ & -0.307214 & -1.931501 & 2.591033 \\
\hline 0 & 2.698664 & 1.041051 & 2.321010 \\
\hline $\mathrm{H}$ & 2.553154 & ๑.992288 & 1.355174 \\
\hline $\mathrm{H}$ & 1.826336 & $\odot .699695$ & 2.591033 \\
\hline 0 & 0.075666 & -1.717461 & -2.910438 \\
\hline $\mathrm{H}$ & 0.744441 & -1.020903 & -3.043994 \\
\hline $\mathrm{H}$ & -0.035506 & -1.705748 & -1.927010 \\
\hline 0 & -1.525198 & 0.793202 & -2.910438 \\
\hline $\mathrm{H}$ & -1.256348 & -0.134253 & -3.043994 \\
\hline $\mathrm{H}$ & -1.459468 & ๑.883623 & -1.927010 \\
\hline 0 & -2.250909 & 1.816586 & 2.321010 \\
\hline $\mathrm{H}$ & -2.135923 & 1.714952 & 1.355174 \\
\hline $\mathrm{H}$ & -1.519122 & 1. 231806 & 2.591033 \\
\hline 0 & 1.449532 & 0.924259 & -2.910438 \\
\hline $\mathrm{H}$ & $\odot .511907$ & 1.155156 & -3.043994 \\
\hline 7 & 1.494974 & ๑. 822125 & -1.92701 \\
\hline
\end{tabular}




\begin{tabular}{|c|c|c|c|}
\hline \multicolumn{4}{|c|}{$\left[\mathrm{SO}_{4}\left(\mathrm{H}_{2} \mathrm{O}\right)_{6}\right]^{2-}\left(C_{2}\right.$ symmetry $)$} \\
\hline $\mathrm{S}$ & $\odot .368137$ & $-\odot .656177$ & $-\odot .555619$ \\
\hline 0 & $\odot .382417$ & $-\odot .661825$ & $\odot .998729$ \\
\hline 0 & 1.803600 & -0.651431 & -1.078739 \\
\hline 0 & $-\odot .380557$ & -1.885042 & $-1.069 \odot 22$ \\
\hline 0 & -0.365463 & 0.631606 & -1.024317 \\
\hline 0 & -2.183659 & -0.512580 & 2.075126 \\
\hline $\mathrm{H}$ & -1.242314 & -0.573883 & 1.772362 \\
\hline $\mathrm{H}$ & -2.576264 & $\odot .062136$ & 1.393412 \\
\hline 0 & 2.162128 & 1.222288 & 2.041976 \\
\hline $\mathrm{H}$ & 2.790621 & 1.260484 & 1.298422 \\
\hline $\mathrm{H}$ & 1.532570 & $\odot .517074$ & 1.748367 \\
\hline 0 & 3.354955 & 1.581630 & -0.682896 \\
\hline $\mathrm{H}$ & 2.857710 & $\odot .745789$ & -0.877920 \\
\hline $\mathrm{H}$ & 2.629406 & 2.233041 & $-\odot .657665$ \\
\hline 0 & -3.122138 & $\odot .488862$ & $-\odot .593057$ \\
\hline $\mathrm{H}$ & -3.229284 & $-\odot .478598$ & $-\odot .636359$ \\
\hline $\mathrm{H}$ & -2.160875 & 0.602835 & $-\odot .800 \odot 83$ \\
\hline 0 & -2.869595 & -2.446749 & -0.049645 \\
\hline $\mathrm{H}$ & -1.973610 & -2.321635 & -0.456429 \\
\hline $\mathrm{H}$ & -2.740401 & -2.035200 & $\odot .825188$ \\
\hline 0 & 0.819761 & 2.943632 & -0.016630 \\
\hline $\mathrm{H}$ & 0.345519 & 2.172356 & -0.418853 \\
\hline $\mathrm{H}$ & 1.092116 & 2.583251 & $\odot .846573$ \\
\hline
\end{tabular}

$\left[\mathrm{SO}_{4}\left(\mathrm{H}_{2} \mathrm{O}\right)_{6}\right]^{2-}\left(C_{I}{ }^{\mathrm{a}}\right.$ symmetry $)$

$\begin{array}{rrrr}\mathrm{S} & 0.025518 & 0.019809 & 0.059631 \\ \mathrm{O} & 0.102588 & 0.039429 & 1.602120 \\ \mathrm{O} & 1.457306 & -0.035432 & -0.515720 \\ \mathrm{O} & -0.691449 & 1.284242 & -0.434014 \\ \mathrm{O} & -0.750915 & -1.230699 & -0.405002 \\ \mathrm{O} & 2.449231 & -2.595481 & -1.304345 \\ \mathrm{H} & 1.822929 & -3.118182 & -0.760174 \\ \mathrm{H} & 2.035489 & -1.704044 & -1.257582 \\ \mathrm{O} & -2.791179 & 0.074970 & -1.977069 \\ \mathrm{H} & -2.308326 & -0.679140 & -1.587211 \\ \mathrm{H} & -2.238904 & 0.780772 & -1.585424 \\ \mathrm{O} & -1.036801 & 2.661415 & 2.055120 \\ \mathrm{H} & -0.627011 & 1.813456 & 2.311921 \\ \mathrm{H} & -1.064997 & 2.489414 & 1.092174 \\ \mathrm{O} & 0.432480 & -3.582962 & 0.497123 \\ \mathrm{H} & 0.834641 & -3.220756 & 1.313190 \\ \mathrm{H} & -0.079905 & -2.803906 & 0.165176 \\ \mathrm{O} & 3.688014 & -1.042130 & 0.816161 \\ \mathrm{H} & 3.580162 & -1.760003 & 0.163309 \\ \mathrm{H} & 2.948394 & -0.457224 & 0.526370 \\ \mathrm{O} & 1.614469 & -2.019887 & 2.656479 \\ \mathrm{H} & 2.439078 & -1.776756 & 2.193061 \\ \mathrm{H} & 1.007532 & -1.293583 & 2.362299\end{array}$




\begin{tabular}{rrrr}
\hline & \multicolumn{3}{c}{$\left[\mathbf{S O}_{\mathbf{4}}\left(\mathbf{H}_{2} \mathbf{O}\right)_{6}\right]^{2-}\left(\boldsymbol{C}_{\boldsymbol{I}}{ }^{\mathbf{b}}\right.$ symmetry $)$} \\
\hline $\mathrm{S}$ & 0.102413 & 0.060616 & 0.011915 \\
$\mathrm{O}$ & 0.099003 & 0.052103 & 1.554801 \\
$\mathrm{O}$ & 1.566050 & 0.008410 & -0.499975 \\
$\mathrm{O}$ & -0.587258 & 1.316898 & -0.506497 \\
$\mathrm{O}$ & -0.636616 & -1.202973 & -0.507398 \\
$\mathrm{O}$ & -1.290294 & 2.597970 & 1.908936 \\
$\mathrm{H}$ & -0.850115 & 1.793823 & 2.240127 \\
$\mathrm{H}$ & -1.181937 & 2.402089 & 0.952816 \\
$\mathrm{O}$ & -0.019981 & -3.546163 & 0.862949 \\
$\mathrm{H}$ & 0.458434 & -3.165870 & 1.629022 \\
$\mathrm{H}$ & -0.329300 & -2.729739 & 0.395269 \\
$\mathrm{O}$ & 3.383181 & -1.804688 & 0.569124 \\
$\mathrm{H}$ & 3.095499 & -2.592741 & 0.068215 \\
$\mathrm{H}$ & 2.772435 & -1.100459 & 0.235119 \\
$\mathrm{O}$ & 1.503819 & -1.996615 & 2.765193 \\
$\mathrm{H}$ & 2.276398 & -1.987036 & 2.164060 \\
$\mathrm{H}$ & 0.974920 & -1.234473 & 2.417630 \\
$\mathrm{O}$ & 1.117993 & -1.829006 & -2.579338 \\
$\mathrm{H}$ & 1.576458 & -1.111650 & -2.090493 \\
$\mathrm{H}$ & 0.279883 & -1.784337 & -2.065062 \\
$\mathrm{O}$ & 2.099660 & -3.988076 & -0.970414 \\
$\mathrm{H}$ & 1.819515 & -3.316338 & -1.630131 \\
$\mathrm{H}$ & 1.365249 & -3.957200 & -0.314688
\end{tabular}




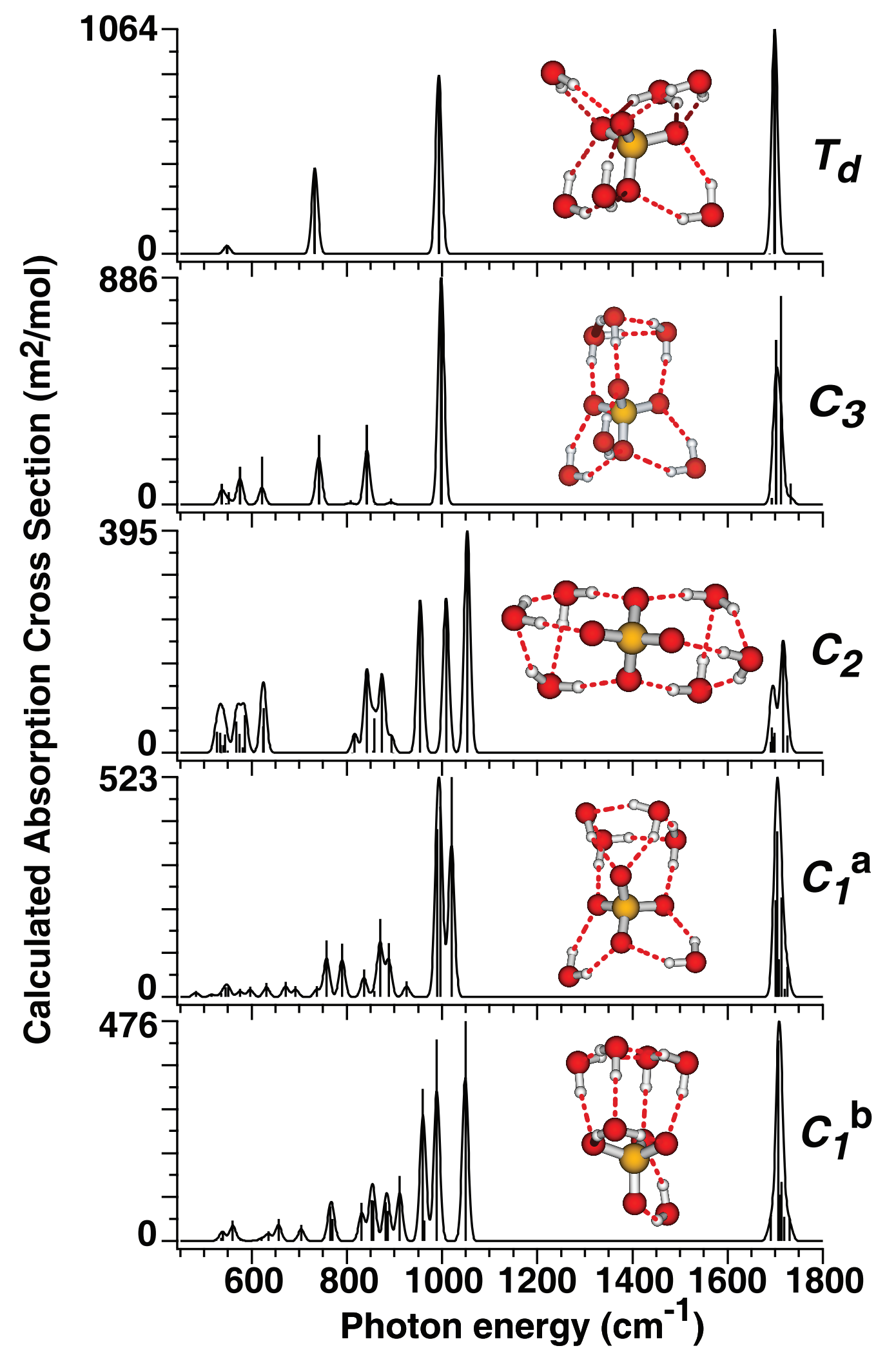

Supplemental Figure 1. Spectra for five low-energy conformers of $\left[\mathrm{SO}_{4}\left(\mathrm{H}_{2} \mathrm{O}\right)_{6}\right]^{2-}$ from B3LYP/AUG-cc-pVDZ calculations. Vibrations are unscaled and have been broadened using $15 \mathrm{~cm}^{-1}$ Gaussian distributions. 
(2a) Squyres, S. W.; Grotzinger, J. P.; Arvidson, R. E.; Bell, J. F.; Calvin, W.; Christensen, P. R.; Clark, B. C.; Crisp, J. A.; Farrand, W. H.; Herkenhoff, K. E.; Johnson, J. R.;

Klingelhofer, G.; Knoll, A. H.; McLennan, S. M.; McSween, H. Y.; Morris, R. V.; Rice, J. W.; Rieder, R.; Soderblom, L. A. Science 2004, 306, 1709-1714.

(2b) Bibring, J. P.; Langevin, Y.; Mustard, J. F.; Poulet, F.; Arvidson, R.; Gendrin, A.; Gondet, B.; Mangold, N.; Pinet, P.; Forget, F. Science 2006, 312, 400-404.

(7) Zhou, J.; Santambrogio, G.; Brümmer, M.; Moore, D. T.; Wöste, L.; Meijer, G.; Neumark, D. M.; Asmis, K. R. J. Chem. Phys. 2006, 125, 11102.

(17) Frisch, M. J.; Trucks, G. W.; Schlegel, H. B.; Scuseria, G. E.; Robb, M. A.; Cheeseman, J. R.; Montgomery, J.; J. A.; Vreven, T.; Kudin, K. N. B., J. C.; Millam, J. M.; Iyengar, S. S.; Tomasi, J.; Barone, V.; Mennucci, B.; Cossi, M.; Scalmani, G.; Rega, N.; Petersson, G. A.; Nakatsuji, H.; Hada, M.; Ehara, M.; Toyota, K.; Fukuda, R.; Hasegawa, J.; Ishida, M.; Nakajima, T.; Honda, Y.; Kitao, O.; Nakai, H.; Klene, M.; Li, X.; Knox, J. E.; Hratchian, H. P.; Cross, J. B.; Bakken, V.; Adamo, C.; Jaramillo, J.; Gomperts, R.; Stratmann, R. E.; Yazyev, O.; Austin, A. J.; Cammi, R.; Pomelli, C.; Ochterski, J. W.; Ayala, P. Y.; Morokuma, K.; Voth, G. A.; Salvador, P.; Dannenberg, J. J.; Zakrzewski, V. G.; Dapprich, S.; Daniels, A. D.; Strain, M. C.; Farkas, O.; Malick, D. K.; Rabuck, A. D.; Raghavachari, K.; Foresman, J. B.; Ortiz, J. V.; Cui, Q.; Baboul, A. G.; Clifford, S.; Cioslowski, J.; Stefanov, B. B.; Liu, G.; Liashenko, A.; Piskorz, P.; Komaromi, I.; Martin, R. L.; Fox, D. J.; Keith, T.; Al-Laham, M. A.; Peng, C. Y.; Nanayakkara, A.; Challacombe, M.; Gill, P. M. W.; Johnson, B.; Chen, W.; Wong, M. W.; Gonzalez, C.; and Pople, J. A. Gaussian 03, Revision B.04; Gaussian, Inc.: Wallingford CT, 2004.

(18) Shin, J. W.; Hammer, N. I.; Diken, E. G.; Johnson, M. A.; Walters, R. S.; Jaeger, T. D.; Duncan, M. A.; Christie, R. A.; Jordan, K. D. Science 2004, 304, 1137-1140. 